I. Boxx, C.D. Carter, M. Stöhr, W. Meier, Study of the mechanisms for flame stabilization in gas turbine model combustors using $\mathrm{kHz}$ laser diagnostics, Experiments in Fluids 54:1532 (2013).

The original publication is available at www.springerlink.com http://dx.doi.org/10.1007/s00348-013-1532-4 


\title{
Study of the mechanisms for flame stabilization in gas turbine model combustors using $\mathrm{kHz}$ laser diagnostics
}

\author{
Isaac Boxx ${ }^{1 *}$, Campbell Carter ${ }^{2}$, Michael Stöhr ${ }^{1}$, Wolfgang Meier ${ }^{1}$ \\ 1: Institute of Combustion Technology, German Aerospace Center, Stuttgart, Germany \\ 2: Air Force Research Laboratory, Wright-Patterson AFB, OH. USA \\ * Isaac Boxx: Isaac.boxx@dlr.de
}

\begin{abstract}
An image-processing routine was developed to autonomously identify and statistically characterize flame-kernel events, wherein $\mathrm{OH}$ (from a planar laser-induced fluorescence, PLIF, measurement) appears in the probe region away from the contiguous $\mathrm{OH}$ layer. This routine was applied to datasets from two gas turbine model combustors that consist of thousands of joint $\mathrm{OH}-$ velocity images from $\mathrm{kHz}$ framerate OH-PLIF and particle image velocimetry (PIV). Phase sorting of the kernel centroids with respect to the dominant fluid-dynamic structure of the combustors (a helical precessing vortex core, PVC) indicates through-plane transport of reacting fluid best explains their sudden appearance in the PLIF images. The concentration of flame-kernel events around the periphery of the mean location of the PVC indicates they are likely the result of wrinkling and/or breakup of the primary flame sheet associated with the passage of the PVC as it circumscribes the burner centerline. The prevailing through-plane velocity of the swirling flow-field transports these fragments into the imaging plane of the OH-PLIF system. The lack of flame-kernel events near the center of the PVC, (in which there is lower strain and longer fluid dynamic residence times), indicates that auto-ignition is not a likely explanation for these flame kernels in a majority of cases. The lack of flame-kernel centroid variation in one flame in which there is no PVC further supports this explanation.
\end{abstract}

\section{Introduction}

The gas turbine (GT) engine is a critical enabling technology for modern aviation and large-scale power generation. Its extensive application, however, has made pollutant emissions a critical factor, and in recent years, a major goal in GT development has been a significant reduction in emissions of oxides of nitrogen $\left(\mathrm{NO}_{\mathrm{x}}\right)$. This goal has lead to considerable interest in the concept of lean premixed- and partially-premixed (LPP) combustion. LPP combustion can produce a homogeneous temperature distribution at the turbine inlet and thus reduced thermal load such that the high peak temperatures of near-stoichiometric conditions responsible for $\mathrm{NO}_{\mathrm{x}}$ production are avoided (Correa, 1998; Lefebvre, 1999). Unfortunately, stabilization of LPP flames is particularly challenging due to their susceptibility to thermo-acoustic instability and blow-out.

The physical mechanism governing flame stabilization and thermo-acoustic pulsation in swirled LPP combustors depends on a complex, interdependent coupling of multiple thermo-physical, chemical and fluid-dynamic parameters. The turbulence-chemistry interaction occurs over a wide range of spatial and temporal scales and is strongly coupled to specific, system-dependent boundary conditions. To better understand the complex dynamics of swirled LPP flames, there has been a sustained effort in recent years to acquire high spatial- and temporal-resolution measurements of multiple combustion parameters in well-characterized, laboratory-scale combustors. This effort has relied extensively on point- and planar-laser diagnostic techniques to measure velocity, major and 
minor species concentrations, their spatial distributions and temperature. Derivative quantities such strain rate and vorticity have also been obtained. Such data, particularly when acquired in combination with acoustic measurements to produce the corresponding phase-averaged values over the thermo-acoustic oscillation, have yielded extensive new insight into the physics of LPP combustion in GT engines. This effort, however, has met with limited success in addressing transient and spatio-temporally unpredictable combustion phenomena such as local extinction, blow-off and auto-ignition. This stems from the relatively low $(\approx 1-10 \mathrm{~Hz})$ repetition rates of standard planar imaging techniques such as particle image velocimetry (PIV) and planar laserinduced fluorescence (PLIF), which prevent one from tracking the temporal development of individual flow structures and their interaction with the flame front.

The recent development of planar measurement systems with multi-kHz acquisition rates and long measurement periods (eg. on the order of one second) have made it possible, for the first time, to statistically characterize spatiotemporally unpredictable phenomena occurring in turbulent flames. Böhm et al. (2008), for example, used simultaneous kHz-PIV and PLIF to study vortexflame interactions leading to local extinction of a turbulent, opposed-jet flame. Steinberg et al. (2010) used similar diagnostics to study flame-hole re-ignition in a turbulent jet flame. Stöhr et al. (2010) used kHz stereo-PIV, OH-PLIF and OH chemiluminescence imaging to study flame blowout in a gas turbine model combustor.

In a series of studies by Boxx et al. (2009a, 2009b, 2010, 2012), multi-kHz framerate stereoscopic-PIV and OH-PLIF were applied simultaneously to study LPP swirl flames in a series of gas turbine model combustors. These measurements revealed the existence of a helical precessing vortex core in the combustor, showed various effects of vortex-flame interaction in the recirculation zone and the impact of thermo-acoustic pulsation on the flow-field and flame dynamics. The data also raised unexpected new questions. Of particular interest were incidents wherein isolated pockets of $\mathrm{OH}$ were observed to suddenly appear in regions of unburned gas upstream of the contiguous reaction zone. We refer to these incidents as 'flame-kernel events'. Flame-kernel events likely arise from one of two physical mechanisms: through-plane convection and propagation of the primary flame sheet, or auto-ignition of unburned gas within the imagingplane. A close examination of point measurements of mixture fraction and temperature acquired in an earlier study via spontaneous Raman scattering in the same burner under identical conditions indicates that auto-ignition may be a viable explanation in some instances. As the measuring system only identifies the presence of $\mathrm{OH}$ and not its source (i.e. transport vs. production), it is impossible to determine conclusively from the PLIF data which explanation is correct for a given instance. If flame-kernel events do stem from auto-ignition, they may represent an important additional mechanism for flame stabilization in swirled flames in GT combustors. The goal of the present study was to statistically characterize the flame-kernel events seen in the Boxx studies (Boxx et al., 2009; Boxx et al., 2010; Boxx, et al. 2012) and to determine the physical mechanism responsible for them.

\section{Experiments}

Data from measurements in two swirl-stabilized gas turbine model combustors were analyzed in this study: the DLR Dual-Swirl (DS) burner and the Turbomeca (TM) burner. Both have been extensively characterized and are well-described in a series of recent publications (GiezendannerThoben et al. 2003, 2005a, 2005b, 2005c; Boxx et al. 2010; Duan et al. 2005; Meier et al. 2007; Boxx et al. 2012). Therefore, only a brief overview of each is presented here.

\section{DS Burner}

The DS-burner consists of two parts: a central nozzle for the introduction of fuel (methane $99.5 \%$ purity) and swirled air into the combustor and an optically accessible combustion chamber. In this burner dry room temperature air enters the combustion chamber through a $15 \mathrm{~mm}$ diameter 
central nozzle. This nozzle is surrounded by a concentric annular nozzle (i.d. $17 \mathrm{~mm}$, o.d. $25 \mathrm{~mm}$ contoured to an o.d. of $40 \mathrm{~mm}$ ). Air enters each nozzle through a set of radial swirl vanes fed from a common plenum. In this study, co-swirl was used. Non-swirling $\mathrm{CH}_{4}$ enters the chamber via a ring of 72 channels $(0.5 \mathrm{~mm} \times 0.5 \mathrm{~mm})$ located between the two air nozzles. The exit planes of the fuel and central air nozzle stand $4.5 \mathrm{~mm}$ below the exit plane of the outer air nozzle. The combustion chamber has a square cross-section measuring $85 \mathrm{~mm} \times 85 \mathrm{~mm}$ and stands $110 \mathrm{~mm}$ tall. The walls of the chamber are quartz plates held at the corners with Inconel ${ }^{\circledR}$ alloy posts (dia. $10 \mathrm{~mm}$ ). The chamber is capped with a steel plate with a central exhaust tube (dia. $40 \mathrm{~mm}$, length $50 \mathrm{~mm}$ ) over a conical contraction.

The flame studied in this work corresponds to that described as 'Flame B' in the work of Weigand et al. (2006). It has a $10 \mathrm{~kW}$ thermal load, corresponding to $12.3 \mathrm{~g} / \mathrm{min}$ of $\mathrm{CH}_{4}$ and $281 \mathrm{~g} / \mathrm{min}$ of air. It has swirl number of 0.55 . Prior studies have shown (Giezendanner et al. 2003, 2005) that this flame displays a strong thermo-acoustic pulsation at $308 \mathrm{~Hz}$ and its flow-field is dominated by a helical precessing vortex core or (PVC) that circumscribes the central axis of the burner at approximately $515 \mathrm{~Hz}$ (Boxx et al. 2010).

\section{TM-Burner}

The TM-burner is a laboratory-scale swirl burner based on an industrial design by Turbomeca S.A. It is operated with a lean $\mathrm{CH}_{4} /$ air mixture at atmospheric pressure. Dry air at room temperature is fed via a $78 \mathrm{~mm}$ diameter plenum into a central nozzle through 12 radial swirl-vanes. Fuel is injected directly into the swirler through twelve $1-\mathrm{mm}$ diameter holes. The exit of the burner nozzle is $27.85 \mathrm{~mm}$ in diameter and has a rounded, conical centerbody. The nozzle exits into a combustion chamber identical to that used by the DS-burner. The injection of fuel (also methane) at the radial swirl vanes results in significant, (albeit imperfect) premixing of the fuel and air prior to their entry into the combustion chamber.

Data from two flame conditions in this burner were analysed in this study: a $25-\mathrm{kW}$ flame with strong self-excited thermo-acoustic pulsations (the 'noisy flame'), and a 30-kW flame that burns stably and without strong thermo-acoustic pulsation (the 'quiet flame'). Both flames were operated with $740 \mathrm{~g} / \mathrm{min}$ of air. The noisy and quiet flames had equivalence ratios of 0.73 and 0.83 (corresponding to 30 and $36 \mathrm{~g} / \mathrm{min} \mathrm{CH}_{4}$ ), respectively. These run conditions were chosen to replicate those studied by Meier et al. (2007) and designated Flame 1 and Flame 2a, respectively (Meier et al., 2007). The same flames were further characterized in Boxx et al. (2010).

\section{Planar Imaging Diagnostics}

The data analyzed in this study were acquired using the DLR $\mathrm{kHz}$ planar measurement system (Boxx et al., 2009a, 2009b, 2010, 2012). This system consists of a planar laser induced fluorescence (PLIF) system for the imaging of the hydroxyl $(\mathrm{OH})$ radical and a stereoscopic particle image velocimetry (PIV) system for the measurement of the 3-component velocity field in a plane. While there were significant differences in the systems used with the DS- and TM-burners, there were strong similarities too. A kHz-rate Edgewave YAG-based laser pumped a Sirah dye laser using rhodamine 590 dye; the dye laser was tuned to the strong $\mathrm{Q}_{1}(7)$ ro-vibronic transition of the $\mathrm{A}^{2} \sum^{+} \leftarrow \mathrm{X}^{2} \Pi$ band $(1,0)$ band. A dual-head Edgewave Nd:YAG laser was used for PIV. Both LIF and particle scattering were collected with CMOS cameras; for LIF, a LaVision HighSpeed IRO intensifier was coupled to the CMOS camera, and a Sodern 45-mm f/1.8 UV lens, fitted with a bandpass filter, collected the fluorescence. Between 4096 and 8192 PLIF-PIV images were recorded for each run. Flame kernel analysis for each condition was based on a single run, i.e. approximately $0.8 \mathrm{~s}$ measurement duration.

The coupled PLIF-PIV system was used to acquire simultaneous planar measurements of the $\mathrm{OH}$ distribution and 3-component velocity field in a plane at 5- and $10 \mathrm{kHz}$ repetition rates for flames in the DS- and TM-burners, respectively. This acquisition rate is insufficient to capture time-resolved 
combustion phenomena at the shortest spatiotemporal scales. Meier et al. (2007), for example, estimate the Kolmogorov scale of the noisy flame in the TM burner to be $0.1 \mathrm{~mm}$, with a corresponding timescale of $35 \mu \mathrm{s}$. The goal of these measurements is acquire time-resolved measurements of turbulence-chemistry interactions occurring at the medium-to-large scales. Prior studies indicate (Boxx et al., 2010; Steinberg et al., 2010; Boxx et al., 2012) that such interactions in the flames are strongly coupled to thermo-acoustic pulsation and the PVC found in these flames. These phenomena occur at approximately $300-500 \mathrm{~Hz}$, and are well-resolved by the acquisition rate of the measurement system. The peak mean velocity magnitude and through-plane velocity of the incoming gases in the flame studied in the DS-burner were measured to be $16.3 \mathrm{~m} / \mathrm{s}$ and $9.7 \mathrm{~m} / \mathrm{s}$ respectively. A fluid element would therefore convect through the field of view of the imaging system in $1.84 \mathrm{~ms}$, or approximately 9 frames. The peak mean velocity magnitude in the TM-burner was $38.6 \mathrm{~m} / \mathrm{s}$, meaning a fluid element would convect though the ca. $35 \mathrm{~mm}$-tall field of view in $0.91 \mathrm{~ms}$, or approximately 9 frames (at $10 \mathrm{kHz}$ ), if it were to stay in the imaging plane that long.

Accuracy of the PIV measurements was $\pm 0.3 \mathrm{~m} / \mathrm{s}$ for the measurements in the DS-burner (Boxx et al., 2010), and $\pm 1.4 \mathrm{~m} / \mathrm{s}$ in the TM-burner (Boxx et al., 2012). The high frame-rate and relatively long measurement duration of the systems enable the acquisition of time-resolved measurements of the flow-field and flame interaction over several hundred thermo-acoustic cycles. The spatial resolution of the PIV was $1 \mathrm{~mm}$ in the DS-burner and $1.1 \mathrm{~mm}$ in the TM-burner. The OH-PLIF measurements in the DS-burner spanned the width of the combustor $(85 \mathrm{~mm})$ and extended $40 \mathrm{~mm}$ downstream of the nozzle exit. For the measurements made in the TM-burner, the PLIF imaging region spanned approximately $50 \mathrm{~mm}$ (wide) $\times 45 \mathrm{~mm}$ (high) and covered slightly more area to the right of the burner centerline than the left.

\section{Image Processing}

The objective of this study was to identify the physical mechanism responsible for the flamekernel events described earlier. In particular, it was to determine the viability of the hypothesis that these $\mathrm{OH}$-concentrations result from auto-ignition of high-temperature fuel-air mixtures away from the primary reaction zone. To accomplish this, image processing tools were developed to automate the identification and statistical characterization of these events. The use of automated feature recognition and statistical analysis tools is essential in dealing with the large datasets generated by the $\mathrm{kHz}$ PLIF and PIV systems. The automated feature recognition tools both sped the identification of flame-kernel events and allowed for a rapid and robust sensitivity analysis of image processing parameters such as the OH-PLIF intensity threshold used to identify a flame kernel.

\section{Flame-Kernel Identification}

Figure 2 shows a typical OH-PLIF image acquired in the DS-burner. The imaging region covers the width of the combustion chamber $(85 \mathrm{~mm})$ and extends approximately $40 \mathrm{~mm}$ downstream of the nozzle. In this image, the regions without $\mathrm{OH}$ (dark) are believed to represent gas at low-tomedium temperatures $(\mathrm{T}<1500 \mathrm{~K})$, e.g. fresh fuel/air mixtures, possibly with some admixture of burned gas. From spontaneous Raman scattering measurements in the same flame, it is known that there is significant mixing of exhaust gas with fresh gas near the nozzle and the gas mixture can cover a wide temperature range $(300-1500 \mathrm{~K})$. The light-grey regions (i.e. those with moderate-tohigh $\mathrm{OH}$ signal levels) within the inner recirculation zone (IRZ) and the outer recirculation zone (ORZ) consist primarily of gas at high temperature $(>1500 \mathrm{~K})$. The brightest regions of the image are indicative of super-equilibrium concentrations of OH. Sadanandan, et al. (2008) showed that, for this flame, the largest magnitude $\mathrm{OH}$-gradients result from super-equilibrium concentrations of $\mathrm{OH}$ and are thus representative of the reaction zone of the flame. Lower magnitude OH-gradients downstream of the contiguous reaction zone tend to be indicative of mixing and decay towards equilibrium.

It is apparent from Figure 2 that most of the measurable concentrations of $\mathrm{OH}$ occur in a 
contiguous region. This region is centered in the IRZ of the burner, but extends to the walls of the combustion chamber and even into the ORZ. At the periphery of this largely contiguous region of $\mathrm{OH}$ is a clearly identifiable interface between burned and unburned gas. The large OH-gradient magnitude associated with this interface indicates a largely intact flame sheet in the burner. Also apparent in Figure 2 are several isolated regions of $\mathrm{OH}$ upstream of the contiguous flame front. These appear in the region of unburned gas several millimetres downstream and to the right of the nozzle exit. Although it is not apparent in this single-frame PLIF image, Figure 3 demonstrates these $\mathrm{OH}-$ concentrations represent flame-kernel events.

\section{Flame -Kernel Extraction}

The extraction of flame-kernel information from the OH-PLIF data occurred in two stages: image filtering / pre-processing and flame-kernel identification. The goal of the first step was to reduce the raw OH-PLIF images to a format wherein isolated flame kernels are readily identifiable by automated checks. The goal of the second step was to apply a series of checks based on physical criteria to identify newly-appearing flame kernels.

\section{Image Processing}

The first step of reducing the raw OH-PLIF images to usable data was the correction for physical parameters such as laser sheet uniformity and camera sensitivity. Correction of the OH-PLIF images for imaging system sensitivity was accomplished by acquiring 1000 images of a uniform illumination lamp. These images were acquired with the same optical configuration and intensifier gain settings used for the PLIF imaging experiments. The ensemble average of these images was then used to correct each PLIF image for camera / intensifier sensitivity. Correction for nonuniformity of the laser-sheet profile was accomplished by acquiring 1000 PLIF images of acetone vapor doped into the combustion chamber. The ensemble-average of these images was similarly used to correct individual OH-PLIF images for laser sheet non-uniformity. The resulting images were then spatially calibrated based on a two-plane calibration target. This calibration eliminates spatial distortions of the imaging objective lens. It also links the spatial coordinates of the corrected OH-PLIF image to that of the combustor and the simultaneously acquired PIV measurement data.

The corrected images were then cropped to show only that region of the combustor illuminated by the laser sheet. The cropped images were then binned $(2 \times 2)$ to one quarter their original size to increase the signal-to-noise ratio, and a low-pass, $5 \times 5$ median filter was applied to smooth the images. The resulting images were binarized based on a user-defined intensity threshold. This threshold was determined empirically, based on how robustly it captures the $\mathrm{OH} /$ unburned-gas interface, as well as the low-intensity regions of $\mathrm{OH}$ in the combustion products further downstream. The binary images were then expanded to their original size for step-two postprocessing.

Step two of the flame-kernel detection algorithm was based on a series of checks of the binary images to identify newly-appearing flame kernels. The first of these checks is designed to eliminate the primary flame front within the image. Using binary image labeling with 8-point connectivity, each contiguous region of $\mathrm{OH}$ in the measurement was identified. Prior knowledge of the flames under study (Boxx et al., 2010) indicates the largest contiguous region of OH in the PLIF images corresponds to the primary flame zone and post-combustion products. The largest labeled region in the binary image was thus assumed to correspond to the primary flame zone and was eliminated.

Next, any regions identified as adjacent to the combustor windows were eliminated. This is based on the assumption that heat-loss there makes auto-ignition unlikely for these flames. Regions of $\mathrm{OH}$ adjacent to the exit plane or the upper edge of the PLIF laser-sheet were also eliminated. At the exit plane, it is impossible to distinguish the in-plane extent of $\mathrm{OH}$-regions due to cut-off of optical access at the burner nozzle. Similarly, the cut-off height of the PLIF laser sheet makes it impossible to rigorously determine the in-plane spatial characteristics of the $\mathrm{OH}$-regions it transects. 
What remains after these checks are binary images that may (or may not) contain one or more flame kernels. Only those $\mathrm{OH}$ concentrations that appear for the first time in the frame represent flame-kernel events. Therefore, binary image labeling was used to identify each region of $\mathrm{OH}$. Each region was then individually checked for overlap with the (complete) binary $\mathrm{OH}$-image from the previous measurement frame. Those showing overlap are assumed to be associated with the $\mathrm{OH}-$ zone of the frame before and thus eliminated from further consideration. Kernels with no overlap of $\mathrm{OH}$ from the previous frame are thus newly-appearing $\mathrm{OH}$ kernels.

The use of OH-PLIF for identification of post-combustion gases in the combustor is sensitive to a number of parameters. These include sensitivity of $\mathrm{OH}$ concentrations to temperature, three-body de-excitation, pulse-to-pulse variation in laser energy and to camera noise. Interpretation of the PLIF images is therefore case-dependent and difficult (or impossible) to accurately reproduce through a synthetic test image. The robustness of the automated image processing tools in the present work was therefore tested against measured PLIF images. Figure 3 shows a series of PLIF images after each stage of the image processing. The first row shows three corrected OH-PLIF images. The second row shows the resulting binary images. The third row shows newly-appearing $\mathrm{OH}$ concentrations identified in step two of the kernel identification algorithm. This figure shows both the robustness of the algorithm and its limitations. The robustness is shown in its ability to both eliminate the primary $\mathrm{OH}$-region and those present in the prior images. Its limitation is in its sensitivity to the choice of intensity threshold used to binarize the images. Although superequilibrium concentrations of $\mathrm{OH}$ present in the reaction zone result in a clearly identifiable interface between burned and unburned gases near the nozzle exit, the lower (equilibrium-level) $\mathrm{OH}$ concentrations in post-combustion products further downstream result in sensitivity to the intensity threshold. The threshold sensitivity is also affected by shot-to-shot variation in pulse energy of the laser. By integrating over the illuminated regions of the acetone PLIF images collected for sheetprofile correction, it was determined the relative pulse energy fluctuation of the $5 \mathrm{kHz}$ PLIF system (used in the experiments on the DS) burner were approximately $\pm 7 \%$ of the mean. The pulse energy fluctuations of the $10 \mathrm{kHz}$ system were of \pm 15 to $20 \%$ of the mean value. As Figure 3 shows, the region affected by the threshold-sensitivity is highly localized (about the burner centerline and approximately $30 \mathrm{~mm}$ downstream) and in a region known to consist almost entirely of postcombustion products. Thus the effect is easily identifiable in the resulting statistics and can therefore be neglected in subsequent analysis.

\section{Kernel Characterization}

Application of the flame-kernel event detection routines described above results in series of binarized images similar to those shown in Row 3 of Figure 3. An automated binary image processing routine was written to scan through the processed images to extract the number of kernels in each frame, their centroid locations and area. The centroid locations were used to determine the local velocity-field characteristics at the flame-kernel events. These data form the basis of the statistics presented below.

It is also important to note the limitations of the resulting statistics. Due to a desire for a PIV resolution of about $1 \mathrm{~mm}$ (equaling the PIV interrogation region size), the field of view of the PIV measurements was significantly smaller than that of the PLIF data in the measurements made in the DS-burner. Therefore, a significant fraction of flame-kernel events occur in regions where no velocity data was available. Statistics relating to local velocity field characteristics are therefore limited to those regions within the measurement window. For the TM-burner, there is almost complete overlap of the PIV and PLIF measurement regions. The field of view, however, does not span the full width of the combustor. The measurement area for the TM-burner was also aligned more to the right side of the burner centerline, biasing the through-plane velocity field statistics toward higher positive values. 


\section{Results and Discussion}

Prior research (Boxx et al., 2010, 2012) indicates the presence of a helical PVC in the flow-field of the DS-burner and in the noisy flame test condition studied in the TM-burner. Proper orthogonal decomposition (POD) analysis has shown the PVC to be a dominant fluid-dynamic structure in these flames. It resides primarily along the shear-layer between the relatively high-velocity jet of incoming reactants and IRZ of the combustor. The passage of the PVC through the combustor flowfield is linked to the phase of the multiplicative constant of the dominant eigenmode of the flowfield. Given its dominant role in combustor dynamics, the PVC likely plays some role in the physical mechanism responsible for flame-kernel events. As such, flame-kernel statistics were sorted into eight bins according to the phase of the PVC, as identified in the multiplicative constant of the first mode of the POD.

Figure 4 shows the phase-sorted probability distribution of flame-kernel events with the DSburner. In this flame, the PVC precesses around the central axis at a frequency of $515 \mathrm{~Hz}$ (Boxx et al., 2010), i.e. with a precession period of $1.94 \mathrm{~ms}$. Thus each phase-average bin corresponds to a timescale of $0.24 \mathrm{~ms}$. Each plot represents approximately 510 measurement frames $\left(1 / 8^{\text {th }}\right.$ of a 4096measurement time series) and contains between 848 and 1030 flame-kernel events. Figure 4 shows that the spatial distribution of flame-kernel events is quite localized and centered in the region of the shear-layer between incoming reactants and the IRZ. This is to be expected, as prior work (Sadanandan et al., 2008) has shown conclusively that the reaction zone of the flame resides primarily in the shear-layer. The thermochemical state of the flame has previously been characterized in the DS-burner by single-shot laser Raman scattering (Meier et al., 2006). A scatterplot of temperature vs. mixture fraction from the region of the shear layer at the heights $5 \mathrm{~mm}$ and $10 \mathrm{~mm}$ is displayed in Fig. 4. The symbols represent the result of the single-shot measurements and the solid line shows the state at adiabatic equilibrium. It is seen that the mixture fraction varies between 0 (pure air) and $\approx 0.15$ (equivalence ratio $\approx 3.1$ ). The samples at $\mathrm{T} \approx 300 \mathrm{~K}$ originate from the inflow of fresh reactants, and the samples with the highest temperatures from the IRZ. The samples with intermediate temperatures are partially reacted mixtures that predominantly represent mixtures of hot gas from the IRZ and fresh reactants. They have not reacted yet because either they have not yet come in contact with a flame front or the residence time was not long enough for auto-ignition. Meier et al. (2006) estimated the ignition delay times for typical compositions found in the shear layer to be below $1 \mathrm{~ms}$ for temperature above $\mathrm{T} \approx 1450 \mathrm{~K}$. However, the estimation did not include effects of turbulence, like scalar dissipation. An estimation of the residence time resulted in a value of $\approx 0.5 \mathrm{~ms}$, of course, with a large scatter due to the turbulent nature of the flow. It was thus concluded that auto-ignition could possibly take place.

Figure 5 shows the spatial distribution of flame-kernel events varies with the phase of the helical PVC. At Phase 1, the distribution peaks approximately $15 \mathrm{~mm}$ to the right and $8 \mathrm{~mm}$ above the origin. A weaker, secondary peak in the distribution is seen on the left side of the combustor. The small cluster of flame-kernel events appearing at the downstream centerline stems from the aforementioned threshold sensitivity in the kernel identification algorithm. Over phases 2 and 3, we see the peak on the right hand side dissipate and the peak to the left increase in magnitude. At phase 4 , the peak on the left side begins to dissipate. This dissipation continues over phases 5 through 8 . At the same time, the peak to the right side grows in magnitude. It is also important to note that axial and radial coordinates of peak flame-kernel concentration on the left and the right side of the combustor also vary with the phase of the PVC. This is consistent with the flame-kernel distribution being coupled with PVC precession, as the PVC itself is (instantaneously) asymmetric about the burner centerline.

Figure 6 shows the phase-averaged velocity field data for the same flame. The color contours represent velocity magnitude. The white lines overlaid are stream-traces. Figure 6 illustrates the effect of the PVC on combustor dynamics. At phase 1 we see a vortex centered approximately $6 \mathrm{~mm}$ to the left and $4 \mathrm{~mm}$ downstream of the nozzle. This vortex lies in the shear-layer between the 
incoming reactants and the IRZ. A second vortex (centered $15 \mathrm{~mm}$ to the left and $28 \mathrm{~mm}$ downstream) is also present. A third vortex is observed approximately $9 \mathrm{~mm}$ to the right of centerline and approximately $13 \mathrm{~mm}$ downstream of the exit. These three vortices represent planar cuts through the helical PVC, which spirals up around the burner centerline, growing in size with downstream distance. Although the field of view of the PIV measurement is insufficient to track these vortices over the entire combustor, it is clear they grow in size and move downstream over a cycle of PVC precession. At phase 4, we see what appears to be a new vortex propagating up into the measurement plane from the swirl nozzle of the combustor. At phase 5, this vortex is fully visible on the right side and the flow-field essentially mirrors that seen at phase 1 . This is to be expected, as prior research (Stöhr et al., 2011b) has shown this phase of the PVC represents its precession half-way around the burner.

The effect of the PVC on the shear-layer between incoming reactants and the IRZ of the combustor is apparent in the velocity magnitude contours plotted in Figure 6. The PVC induces significant distortion of the shear-layer as it precesses through the combustor. As much of the reaction zone in this flame resides in the shear-layer, this PVC-induced distortion can be expected to significantly impact flame sheet wrinkling and curvature. Indeed, evidence of this has been reported in prior studies on the flame (Steinberg et al., 2011). Figure 7 shows the same phaseaveraged data overlaid on the Vz-component of velocity. It is clear from this figure that the precession of the PVC does not induce a major disruption in the through-plane component of velocity.

Comparison of Figures 5,6 and 7 reveals a number of clues as to the physical mechanism responsible for the flame-kernel events. It was noted above that the concentration of flame-kernel events at phase 1 occurs at $(\mathrm{x}, \mathrm{y}) \approx(15 \mathrm{~mm}, 8 \mathrm{~mm})$. Inspection of the phase-averaged velocity field data in Figure 6 shows that this point stands at the outer periphery of one of the vortices identified as representing the PVC. Indeed, the peak concentration of flame-kernel events at this phase of the PVC precession is found almost directly between the center of the PVC and the high-velocity jet of incoming reactants. The remaining plots in Figures 5-7 reveal similar correlation for each phaseangle. At phase 6 , for example, the peak flame-kernel concentration is observed at $(\mathrm{x}, \mathrm{y}) \approx(9 \mathrm{~mm}$, $7 \mathrm{~mm}$ ). It is clear from Figure 6 that this point is also at the periphery of the PVC (well away from its center) and within the high-velocity shear layer between incoming reactants and the IRZ. No significant difference in the through-plane velocity field is observed at this location at phase 6 in Figure 7.

The concentration of flame-kernel events in the shear-layer around the outer-periphery of the mean PVC location means the flame kernels appear most frequently in a region less conducive to auto-ignition than just a short distance away, at the center of the PVC, where residence times are longer and strain-rates are lower. The spatial location of peak flame-kernel concentration is, however, highly conducive to flame wrinkling and strain-induced local extinction. Given these observations, it appears flame-kernel events are likely the result of increased flame-sheet wrinkling and breakup induced by the passage of the PVC through the combustor. Fragments or tendrils of the flame sheet resulting from this interaction are transported into the PLIF imaging plane by the prevailing through-plane velocity of the swirling flow in what we identify as flame-kernel events. Such an interpretation is consistent with the findings of Stöhr et al. (2009, 2011b), wherein PLIF images acquired in a plane oriented horizontal with respect to the swirl nozzle showed significant wrinkling of the flame sheet associated with the passage of the PVC.

Figure 8 shows the phase-sorted probability distribution of flame-kernel events for the noisy flame condition measured in the TM-burner. In this flame, the PVC precesses around the central axis at a frequency of $570 \mathrm{~Hz}$ (Boxx et al., 2012), i.e. with a precession period of $1.75 \mathrm{~ms}$. Thus each phase-average bin corresponds to a timescale of $0.219 \mathrm{~ms}$. Despite the fact that the OH-PLIF images in this case do not span the entire width of the combustor, a similar correlation between the spatial distribution of flame-kernel events and the passage of the PVC is observed. In this case, at phase 1 
the distribution of flame-kernel events occurring to the right of centerline peaks at $(\mathrm{x}, \mathrm{y}) \approx(18 \mathrm{~mm}$, $25 \mathrm{~mm}$ ). Figure 9 shows the corresponding phase-averaged velocity fields. Here again, we see the peak concentration of flame-kernel events occurs in the shear-layer region at the outer periphery of the vortex centered at $(\mathrm{x}, \mathrm{y}) \approx(12 \mathrm{~mm}, 20 \mathrm{~mm})$. The spatial distribution of flame-kernel events is considerably broader in this case compared to that seen in the DS-burner and located further from the swirl nozzle. This is consistent with the considerably larger area over which the vortex / shearlayer interaction takes place in this flame. Here again, we see little evidence of a significant disruption of the through-plane velocity field by the PVC. Inspection of the flame-kernel event distributions at each subsequent phase shows they consistently peak in the shear-layer region at the outer periphery of the PVC. The similarity in peak flame-kernel event concentration despite significant differences in fuel-air mixing characteristics between the two burners is also consistent with a fluid-dynamic mechanism (i.e. flame-wrinkling and breakup induced by the PVC traversing the shear-layer) rather than a thermo-chemical (i.e. auto-ignition) mechanism being responsible for the flame-kernel events.

Figure 10 shows the distribution of flame-kernel events measured in the 'quiet flame' in the TMburner, phase-sorted according to the dominant velocity field eigenmode. As with the noisy flame, the left-right asymmetry observed in this figure stems from the positioning of the measurement region relative to the burner centerline. Consistent with the results seen for the noisy flame and in the DS-burner, flame-kernel events are concentrated in the shear-layer between incoming reactants and the IRZ. Indeed, the peak concentration of flame-kernel events is higher with the quiet flame than in either of the other two cases. The results are unique, however, in that the peak concentration remains virtually unchanged when sorted with respect to the dominant eigenmode of the velocity field. The axial location of peak flame-kernel event concentration varies with the phase of the dominant eigenmode, but its magnitude remains largely constant. Figure 11 shows the corresponding phase-averaged velocity fields for this case. Consistent the previous two cases, peak concentration of flame-kernel events occurs in the shear-layer at the periphery of a large vortex.

Prior research has shown (Boxx et al., 2012) that no PVC exists in the combustor flow-field for the 'quiet flame' in the TM-burner. The dominant velocity-field eigenmode is representative of a resonant acoustic mode of the combustor. This mode has a frequency of $364 \mathrm{~Hz}$ (Boxx et al., 2010), and therefore a period of $2.75 \mathrm{~ms}$. Each phase-average bin corresponds to a timescale of $0.343 \mathrm{~ms}$. The periodic fluctuation seen in this POD mode is linked to the (very weak) self-excited thermoacoustic pulsation of this flame. The vortices seen in the phase-averaged velocity field in this case represent shear-layer vortices shed at the combustor exit that propagate up through the flow. A weak, self-excited thermo-acoustic pulsation at this resonant frequency helps drive the propagation of these vortices as they move through the shear-layer.

The results shown in Figures 10 and 11 are consistent with vortex-induced flame wrinkling and breakup being the dominant mechanism responsible for flame-kernel events observed in this combustor. In this case however, it is shear-layer vortices that induce this wrinkling and breakup rather than a PVC. The absence of a PVC for this flame condition results in more circumferentially uniform shear-layer vortices. This explains the lack of phase-dependence in the peak concentration of in-plane flame-kernel events. The axial variation of peak flame-kernel concentration is consistent with axial velocity fluctuations induced with thermo-acoustic pulsation. Finally, the fact that peak concentration of flame-kernel events consistently appears approximately $20 \mathrm{~mm}$ downstream of the nozzle is explained by the fact that shear-layer vortices grow with downstream distance. In the nearnozzle regions, they have yet not reached sufficient size to induce significant flame-surface wrinkling or breakup.

Although the phase-sorted probability distributions of flame-kernel events in the DS-burner and the noisy flame of the TM-burner indicate a clear correlation with the passage of the PVC, much instantaneous data is lost in the phase-averaging process. Given the spatiotemporal 'blurring' of statistics caused by phase-averaging, it is plausible to suggest that a significant fraction of the 
flame-kernel events could be associated with strong local fluctuations in one or more flow-field quantities caused by 'outlier' events in the flow not related to the dominant flow-field structure (the PVC). It is therefore important to ascertain how faithfully the phase-averaged quantities capture the behavior of individual flame-kernel events. Of particular interest in the present work is whether the instantaneous strain-rate at the flame kernel location is well captured by the phase-averaged field. This was checked by computing the $2 \mathrm{D}$ minimum normal strain at the centroid location of each flame kernel identified in the overlapped PIV / PLIF measurement plane and subtracting its phaseaveraged value. Figure 12 presents histograms showing the distributions of these values at four phases of the flame measured in the DS-burner. This figure clearly shows that at all phases, the histograms have a strong peak at zero, with an approximately symmetric distribution about the zero value. Similar results were measured in the noisy flame of the TM-burner. The fact the histograms peak at zero demonstrates that the local strain-rate at most flame-kernel events is approximately equal to the phase-averaged value at the same location. One may therefore conclude that the majority of flame-kernel events are not the result of strong fluctuations in the flow occurring independently of the PVC. That is, they are not frequently associated with 'outlier' events in the flow-field statistics. This finding is consistent with the previous conclusion that flame-kernel events are likely the result of flame-wrinkling and breakup induced by the passage of the PVC.

Taken together, the data from the DS- and TM-burners indicate auto-ignition is not the dominant mechanism responsible for the flame-kernel events observed in the $\mathrm{kHz} \mathrm{OH-PLIF} \mathrm{data.} \mathrm{Although}$ the data does not exclude the possibility that some flame kernels result from auto-ignition, the majority of flame-kernel events occur in regions of the flow not highly conducive to auto-ignition. Were auto-ignition the dominant mechanism responsible for the observed flame-kernel events, one would expect a greater concentration of them in regions of the flow such as at the core of the PVC, where strain-rates are lower and residence times are longer. The concentration of flame-kernel peaks in all cases at the interface between the dominant vortex structure (the PVC in two cases, the shear-layer vortices in the third) and the shear-layer indicates that the mechanism responsible is wrinkling and breakup of the primary flame sheet in the combustor. In this mechanism, the sudden appearance of flame-kernels in the PLIF images is explained by through-plane transport of the highly-wrinkled or fragmented flame sheet.

\section{Conclusions}

The application of $\mathrm{kHz}$ framerate planar laser-induced fluorescence (PLIF) of the $\mathrm{OH}$ radical and stereo particle image velocimetry (PIV) to a series of lean, partially-premixed swirl flames in a pair of gas turbine model combustors revealed a phenomenon we refer to as flame-kernel events, defined as the sudden appearance of a region of high $\mathrm{OH}$-concentration fluid in the measurement plane of the OH-PLIF imaging system away from the contiguous flame front. Prior research suggests flame-kernel events may represent auto-ignition of hot, unburned fuel-air mixtures in the combustor. If confirmed, this would represent a new and potentially important mechanism for flame stabilization and control in swirled LPP flames. As flame-kernel events are spatio-temporally unpredictable, they are identifiable only in time-resolved sequences of planar measurements such as $\mathrm{kHz}$ framerate OH-PLIF. Given the large (several thousand frames per imaging run) datasets acquired in this study and the unpredictability of where flame-kernel events will occur, the use of automated feature recognition and statistical analysis tools was required. The objective of this study was to develop such tools and to use them to test the hypothesis that flame-kernel events represent auto-ignition through rigorous statistical analysis.

An image-processing routine was developed to autonomously identify and statistically characterize the flame-kernel events. Phase sorting of the kernel centroids with respect to the dominant fluid-dynamic structure of the combustors (a helical precessing vortex core or 'PVC') revealed a strong correlation between flame-kernel events and the precession of the PVC. The concentration of flame-kernel events around the periphery of the mean location of the PVC 
indicates these events are likely caused by wrinkling and breakup of the primary flame sheet associated with the passage of the PVC as it circumscribes the burner centerline. The prevailing through-plane velocity of the swirling flow-field transports these fragments into the imaging plane of the OH-PLIF probe. The lower concentration of flame-kernel events near the center of the PVC, (where there is lower strain and longer fluid dynamic residence times), suggests auto-ignition is not a likely explanation for these flame kernels in a majority of cases. The lack of flame-kernel centroid variation in the one flame in which there is no PVC further supports this explanation.

\section{Acknowledgements}

This work was supported by the Air Force Office of Scientific Research (AFOSR) and The European Office of Aerospace Research \& Development (EOARD) through Grant FA8655-10-1-3052. Christoph Arndt participated in one of the measurement campaigns that produced data analyzed in the present study.

\section{References}

R. Barlow. Proc. Comb. Inst. (2007), 49-75.

B. Böhm, I. Boxx, C. Kittler, W. Meier, A. Dreizler. Proc. Combust. Inst. 32 (2009) 1647-1654

Boxx I, Heeger C, Gordon R, Böhm B, Dreizler A, Meier W (2009b) Combust. Flame, 156:269-271.

I. Boxx, M. Stöhr, C. Carter, W. Meier. Applied Physics B, 95(1) 2009a. 23-29.

I. Boxx, M. Stöhr, C. Carter, W. Meier. Combustion and Flame 157(8) (2010) 1510-1525. doi:

10.1016/j.combustflame.2009.12.015.

I. Boxx, C. Arndt, C. Carter, W. Meier. Exp. Fluids, 52:3 (2012) 555-567. 2012. DOI: 10.1007/s00348-010-1022-x

S. Correa. Proc. Comb. Inst. (1998) 1793-1807.

X. R. Duan, W. Meier, P. Weigand, B. Lehmann, Appl. Phys. B 80 (2005) 389-396.

R. Giezendanner-Thoben, O. Keck, P. Weigand, W. Meier, U. Meier, W. Stricker, M. Aigner, Combust. Sci. Tech. 175 (2003) 721-741.

R. Giezendanner-Thoben, P. Weigand, X. R. Duan, W. Meier, U. Meier, M. Aigner, B. Lehmann, J. Eng. Gas Turb. Power. 127 (2005a) 492-496.

R. Giezendanner-Thoben, U. Meier, W. Meier, M. Aigner. Flow Turb. Combust. 75 (2005b) 317-333.

R. Giezendanner-Thoben, U. Meier, W. Meier, J. Heinze, M. Aigner, Appl. Opt. 44 (2005c) 6565-6577

A.H. Lefebvre, Gas Turbine Combustion, Taylor \& Francis, Philadelphia, 1999.

W. Meier, X.R. Duan, P. Weigand. Comb. Flame. 144 (2006) 225-236.

W. Meier, P. Weigand, XR Duan, R. Giezendanner-Thoben. (2007) Combust. Flame 150:2-26.

doi.org/10.1016/j.combustflame.2007.04.002

R. Sadanandan, M. Stöhr, W. Meier. Appl. Phys. B 90 (2008) 609-618

A. M. Steinberg, I. Boxx, C. Arndt, J. Frank, W. Meier. Proc. Combust. Inst. 33 (2011) 1663-1672.

A. Steinberg, I. Boxx. M. Stöhr, C. Arndt, W. Meier, C. Carter. AIAA-2011-5554. 47th AIAA/ASME/SAE/ASEE Joint Propulsion Conference and Exhibit, San Diego, California, July 31-3, 2011

M. Stöhr, R. Sadanandan, W. Meier. Proc. Combust. Inst. 32. (2009) 2925-2932.

M. Stöhr, I. Boxx, C. Carter, W. Meier. Proc. Combust. Inst. 33 (2011) 2953-2960.

M. Stöhr, R. Sadanandan, W. Meier. Exp Fluids 51 (2011b) 1153-1167

P. Weigand, W. Meier, X. R. Duan, W. Stricker, M. Aigner. Combust. Flame 144 (2006) 205-224. 


\section{Figures}

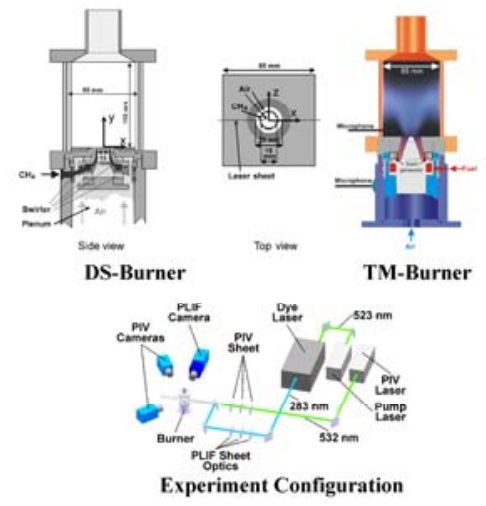

Figure 1 - Burners and experiment configuration

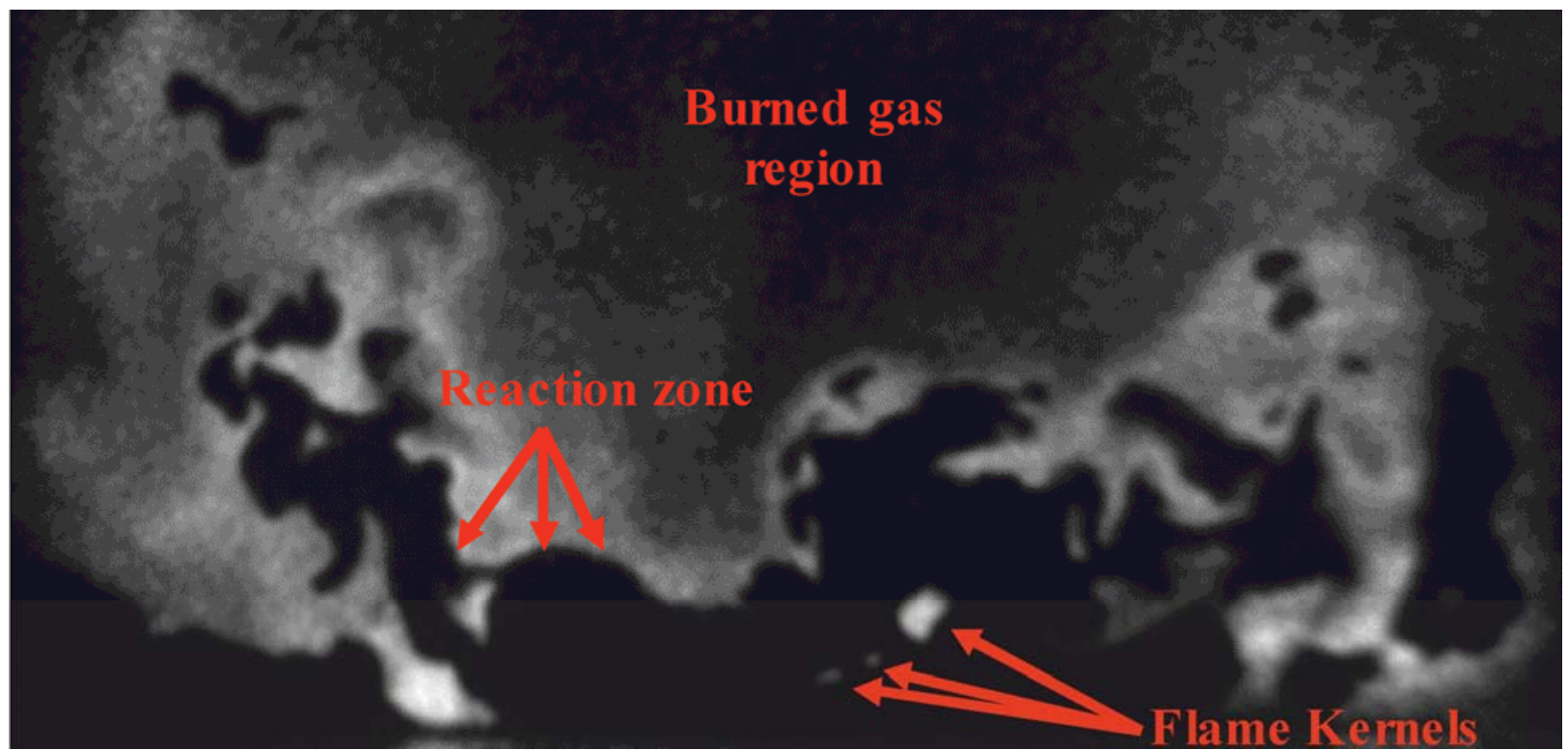

Figure 2 - Representative OH-PLIF Image 

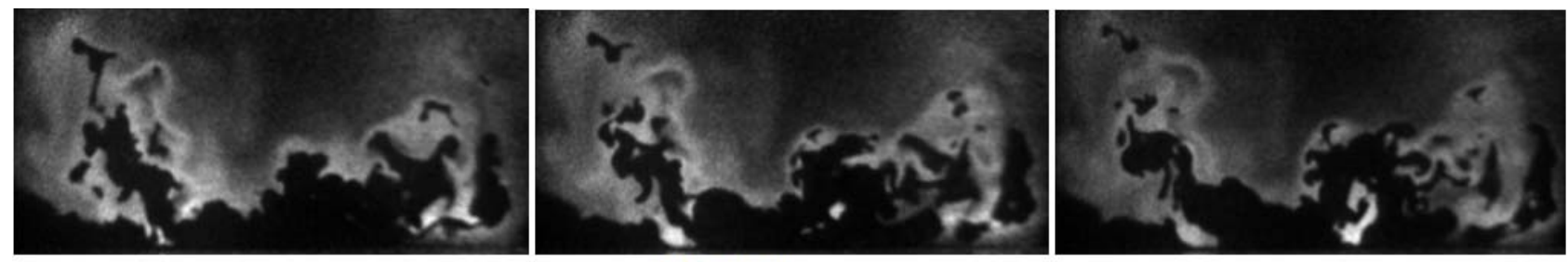

PLIF Images

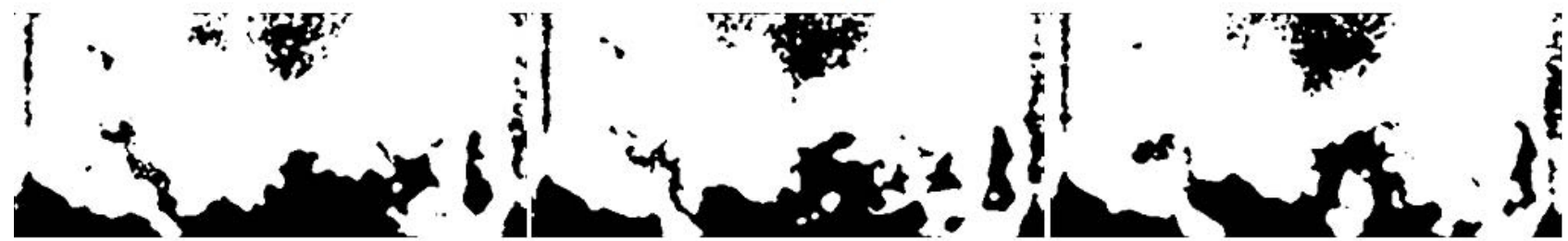

Binarized Images
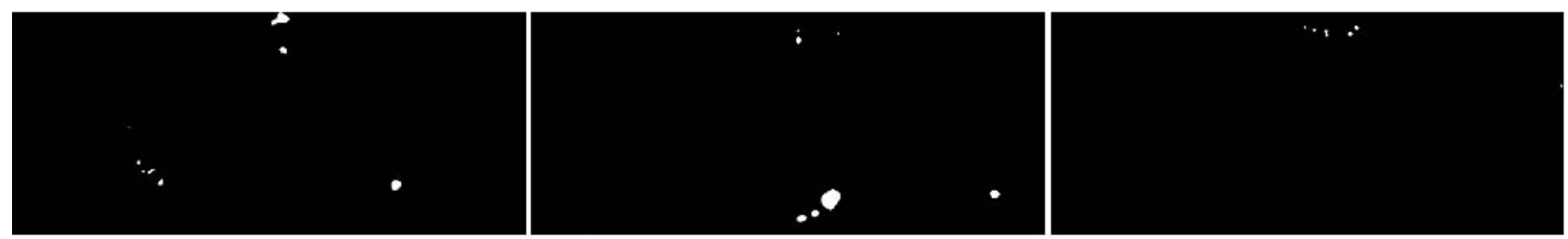

With Feature Recognition

Figure 3 - Steps in the flame-kernel event identification algorithm.

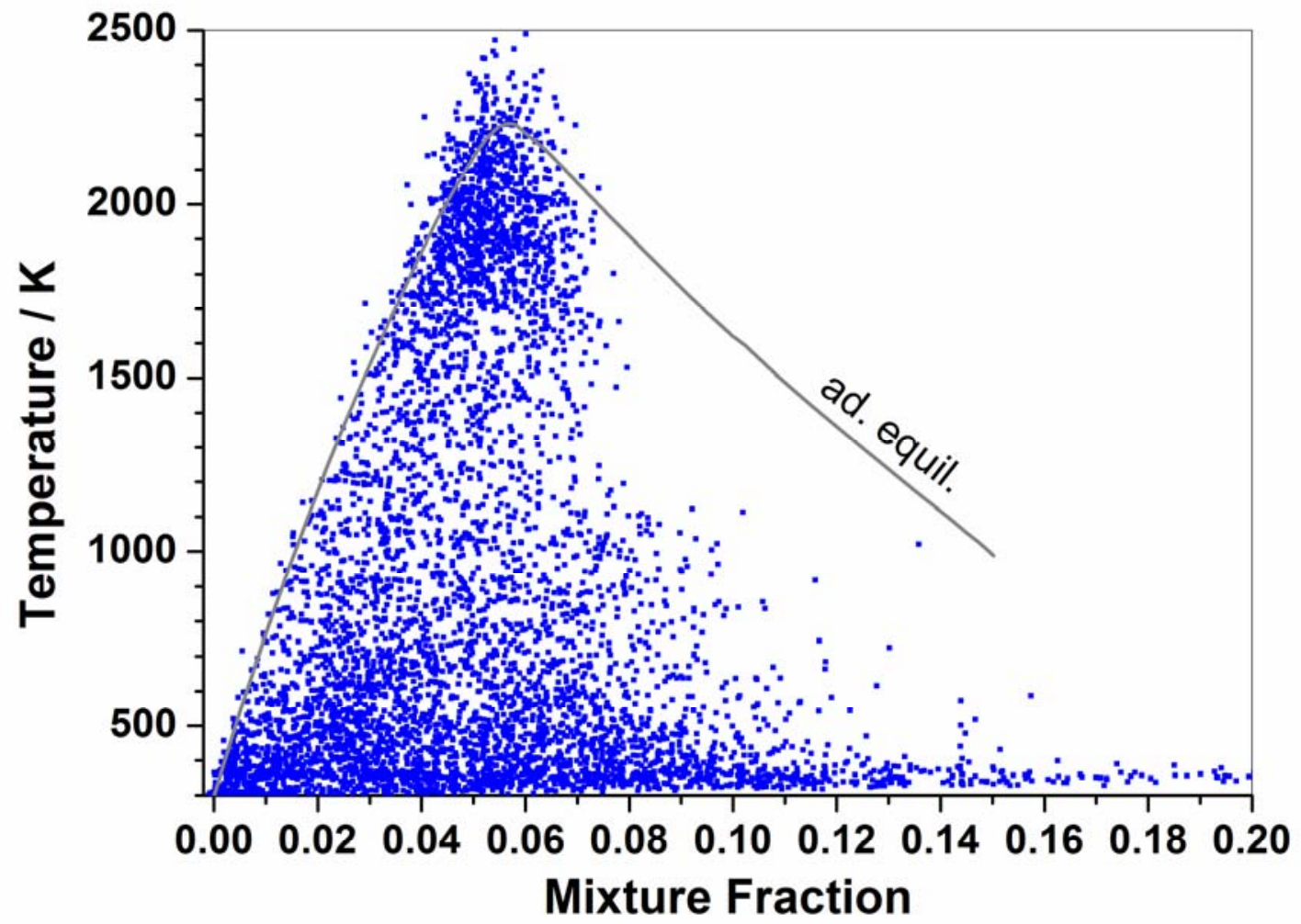

Figure 4 - Raman measurements of temperature and mixture fraction in the shear-layer region $(\mathrm{h}=$ $5 \& 10 \mathrm{~mm}, \mathrm{r}=5-21 \mathrm{~mm}$ ) of the DS-burner. (Originally presented in Meier et al., 2006). 

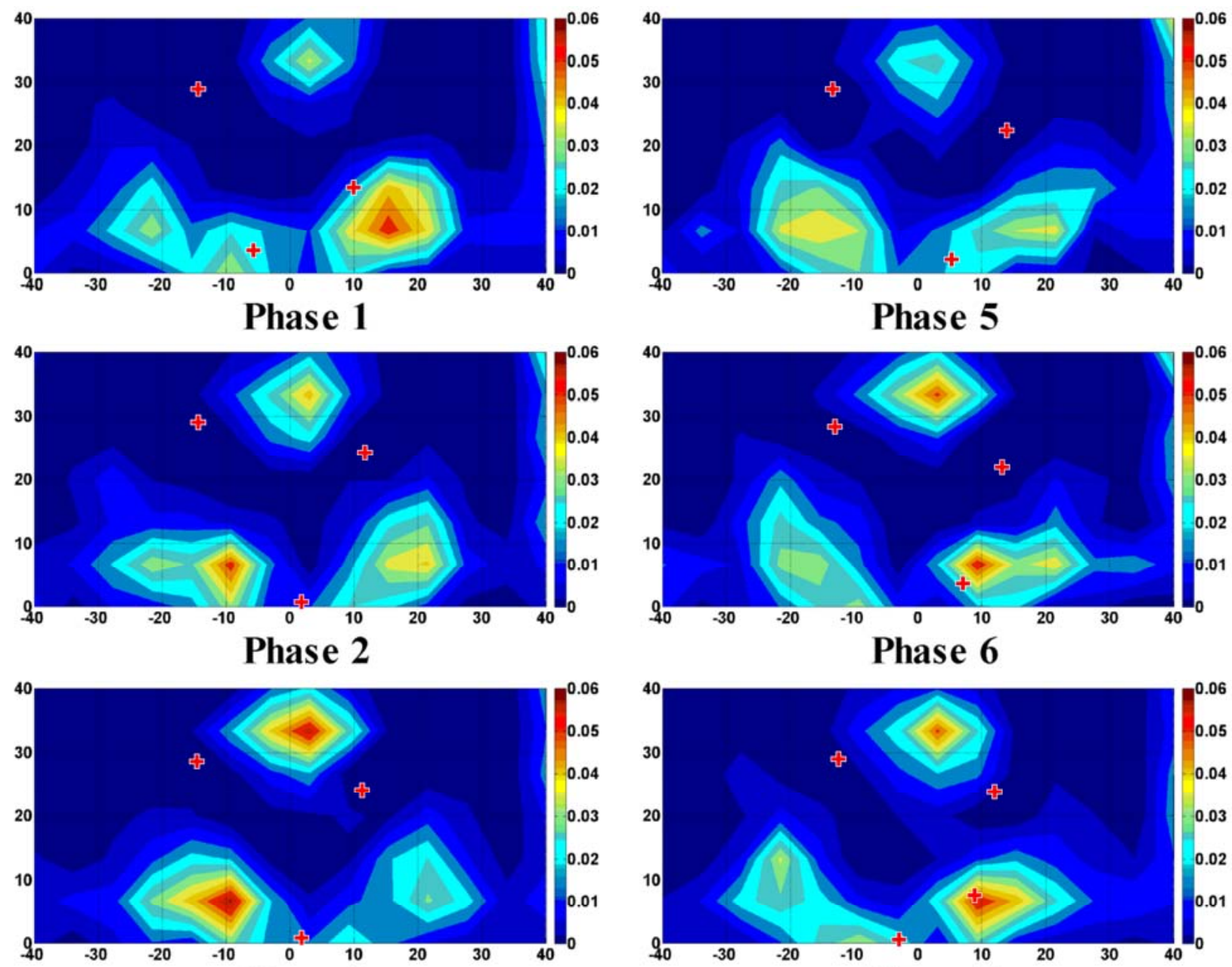

Phase 3

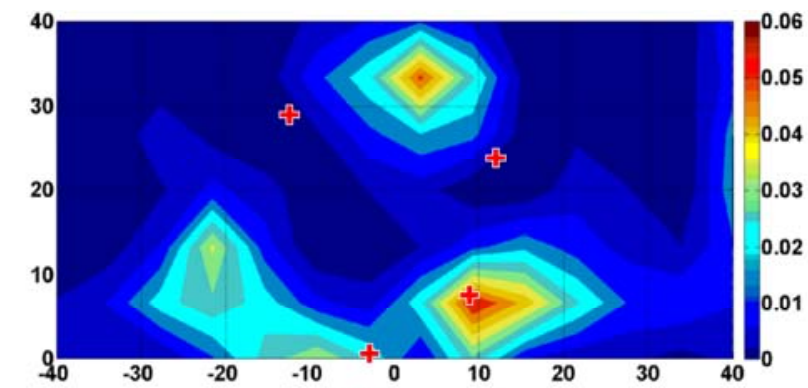

Phase 7
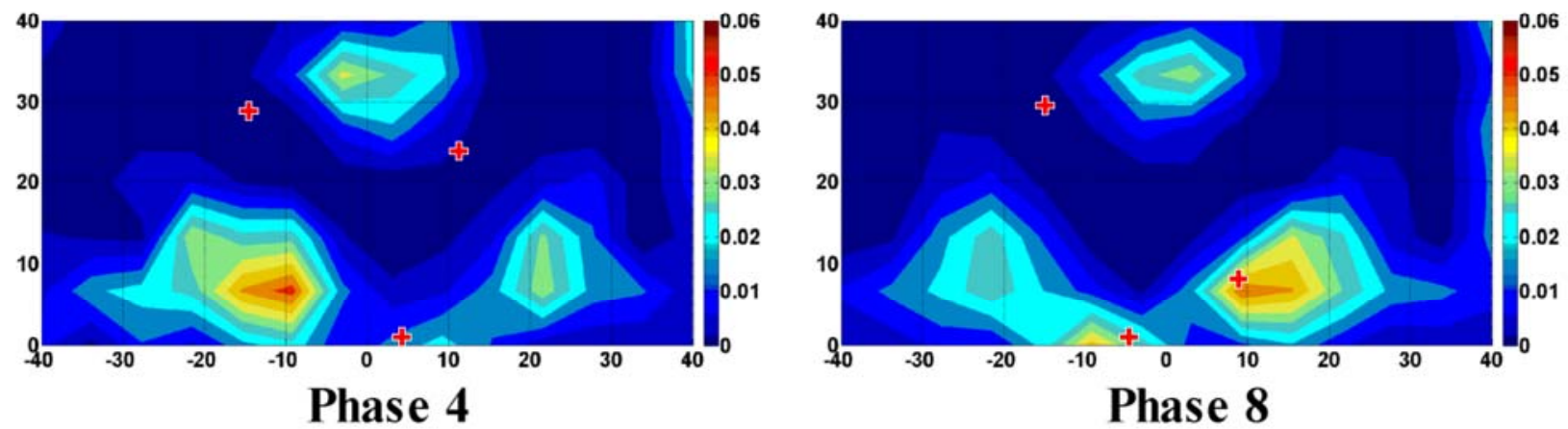

Figure 5 - Phase-sorted probability distribution of flame-kernel events in the DS-burner. The red crosses indicate the corresponding PVC center location at each phase angle. 

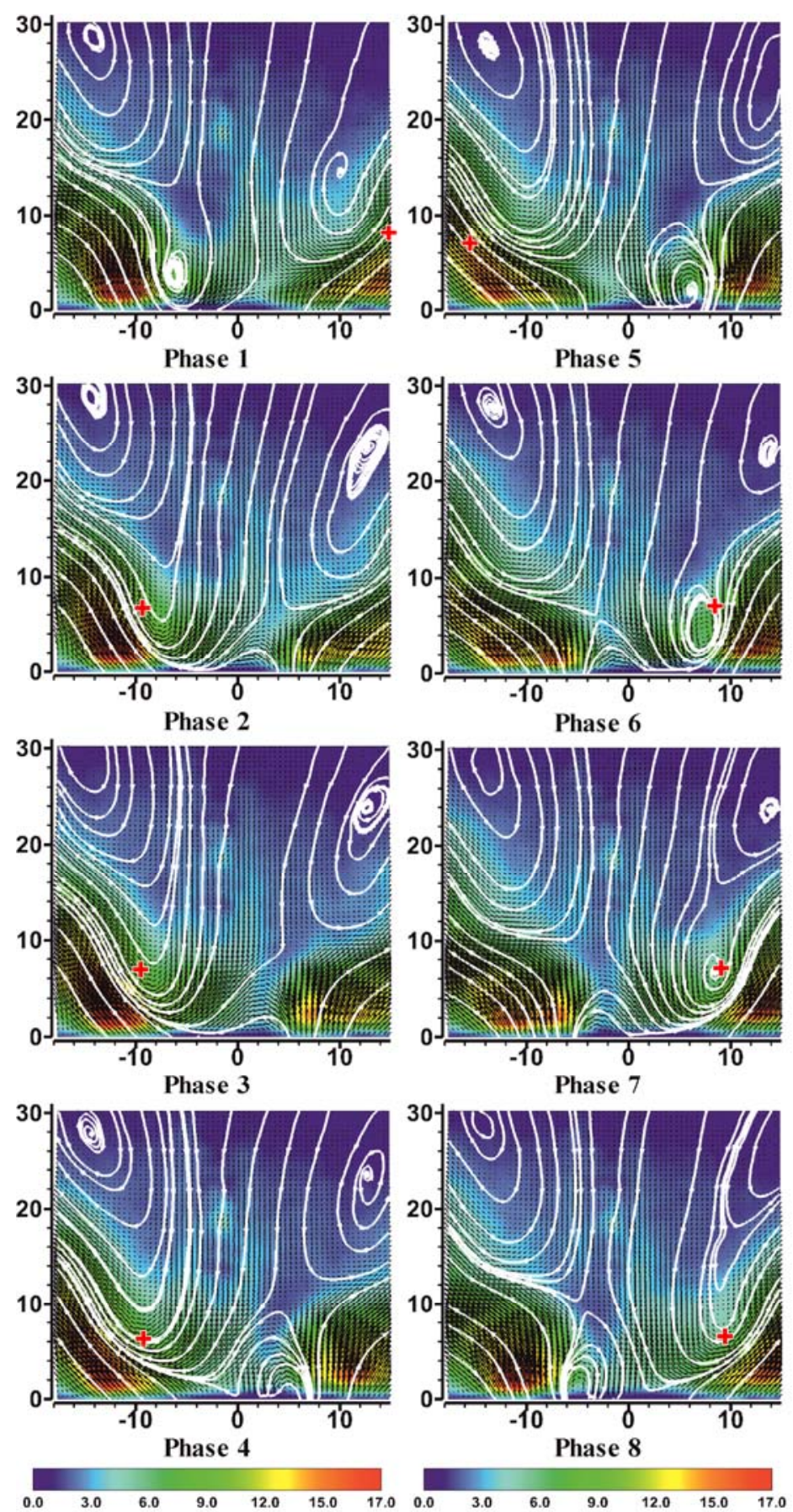

Figure 6 - Phase-averaged velocity magnitude distribution in the DS-burner. The red crosses indicate peak flame-kernel densities from corresponding phase angle. 

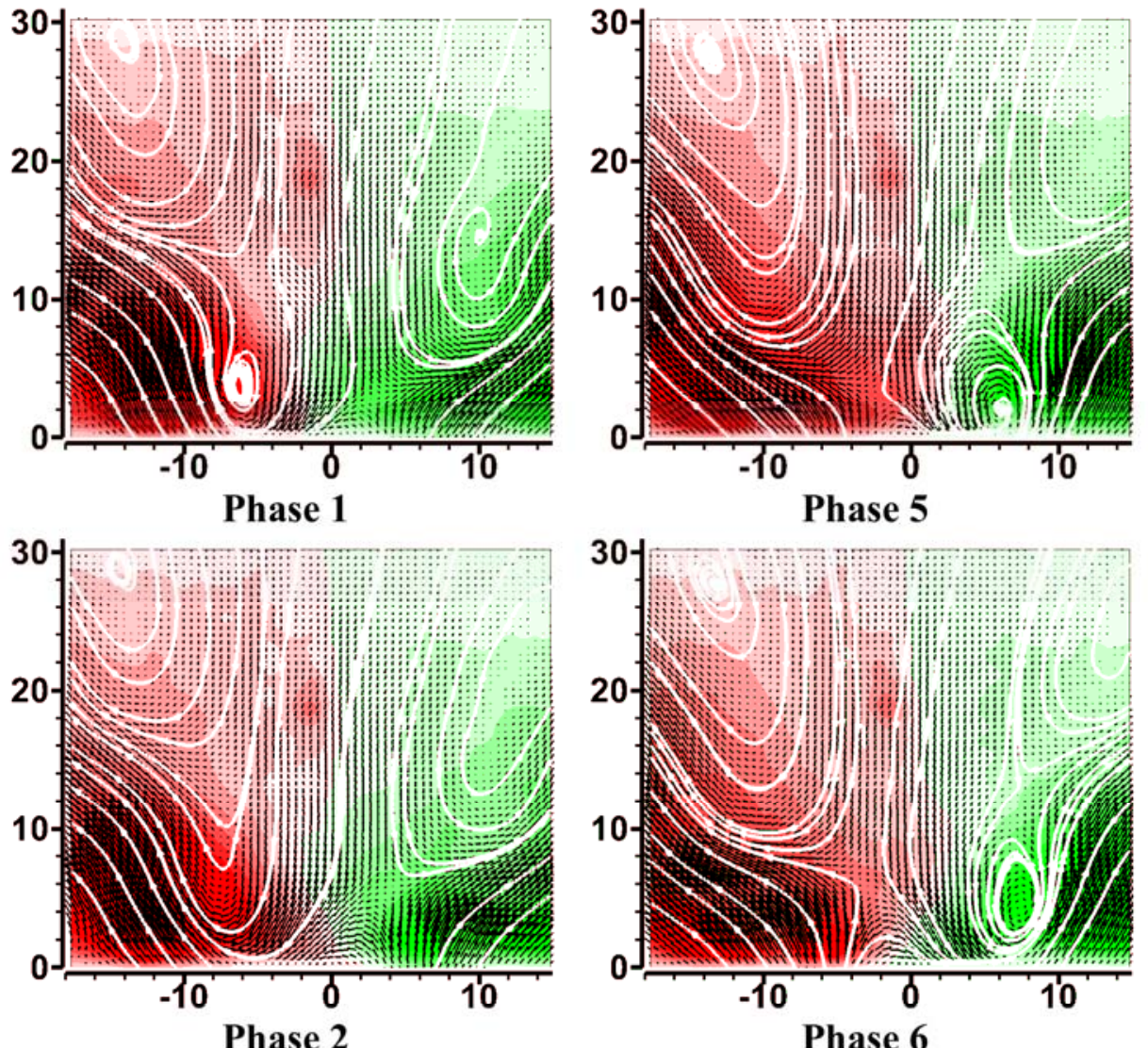

Phase 5
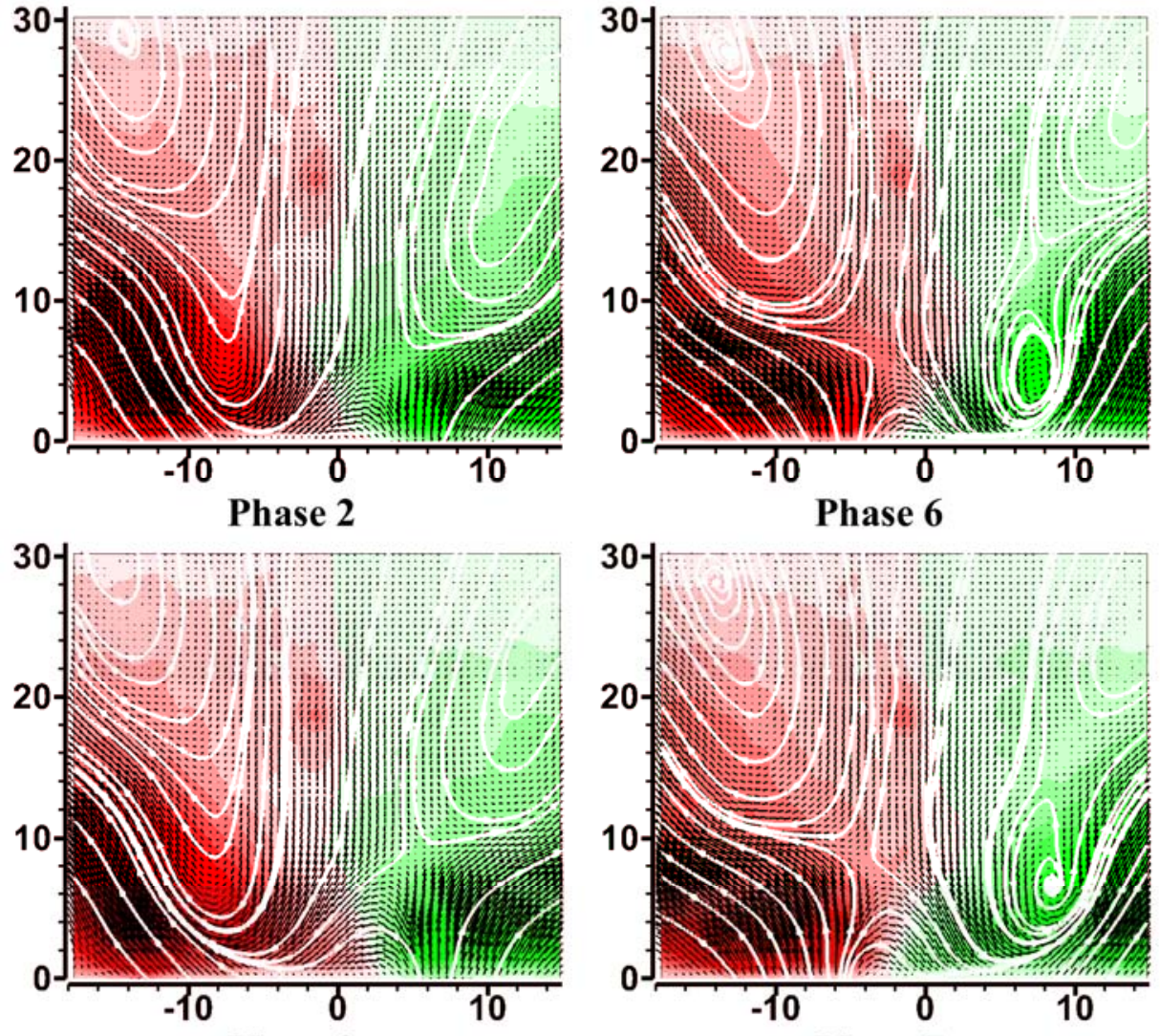

Phase 3

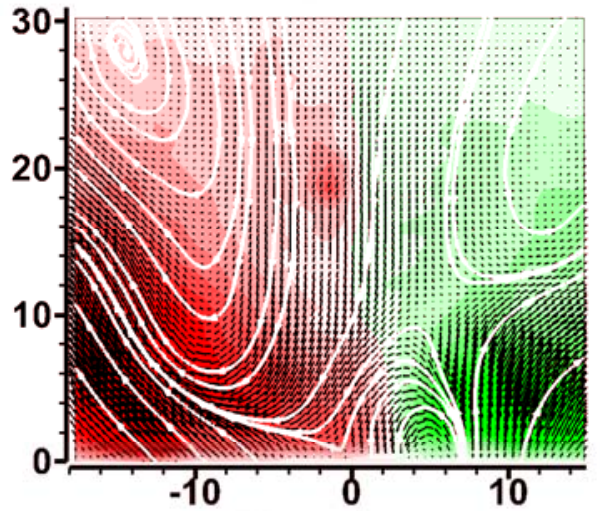

Phase 4

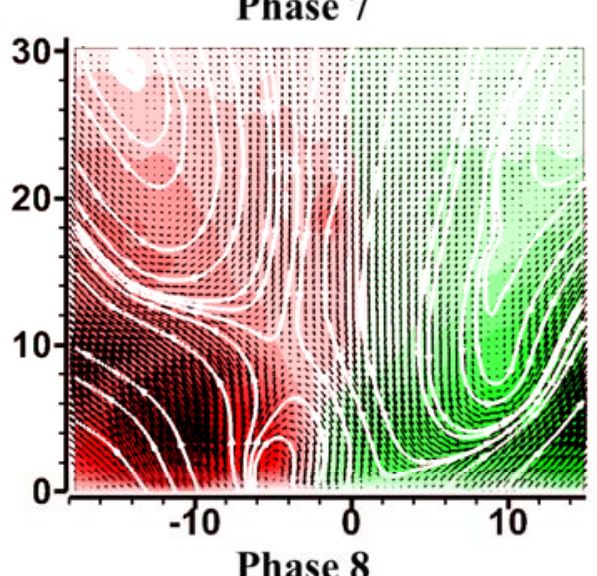

$\begin{array}{lllllllllllll}-6 & -5 & -4 & -3 & -2 & -1 & 0 & 1 & 2 & 3 & 4 & 5 & 6\end{array}$

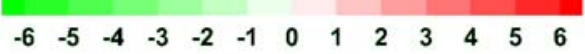

Figure 7 - Phase-averaged through-plane velocity in the DS-burner. 


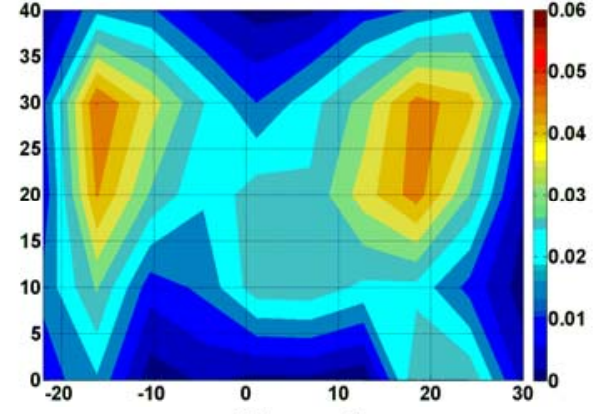

Phase 1

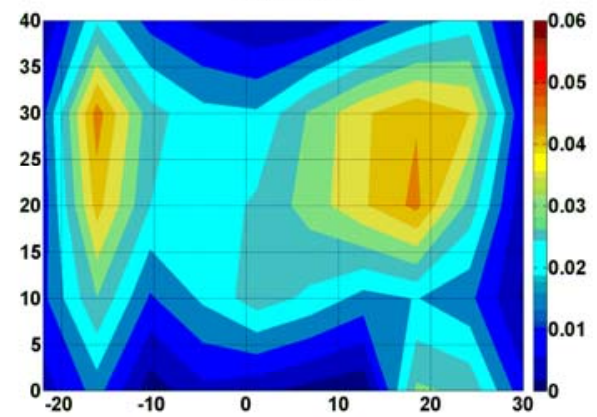

Phase 2

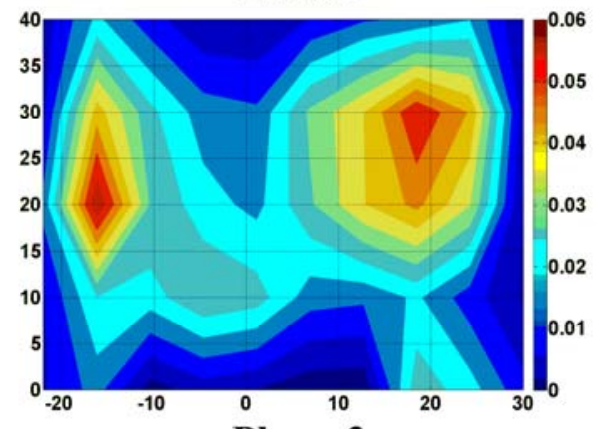

Phase 3

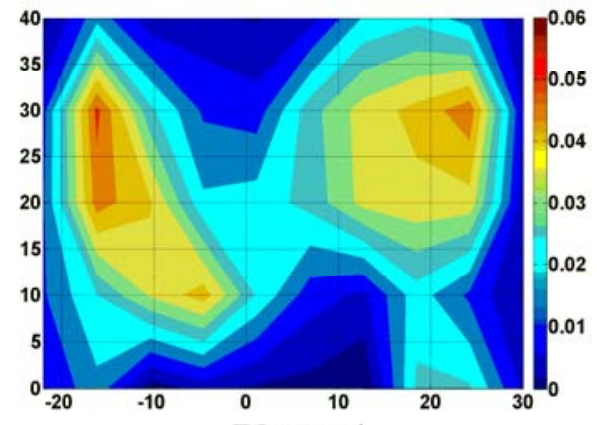

Phase 4

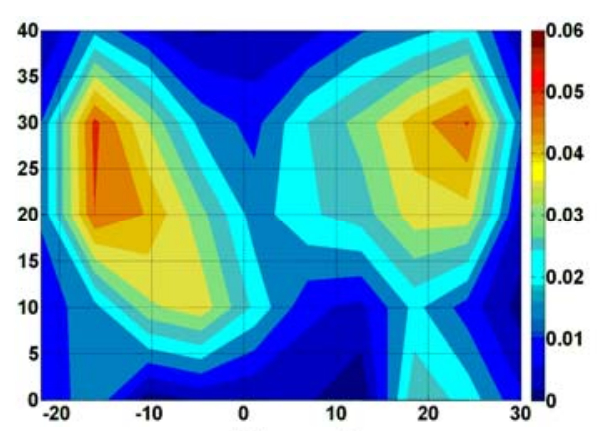

Phase 5

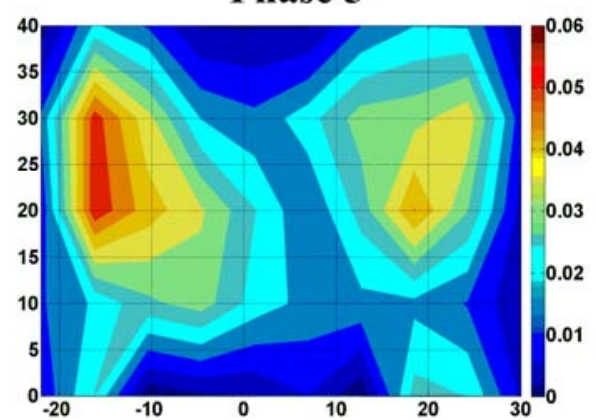

Phase 6

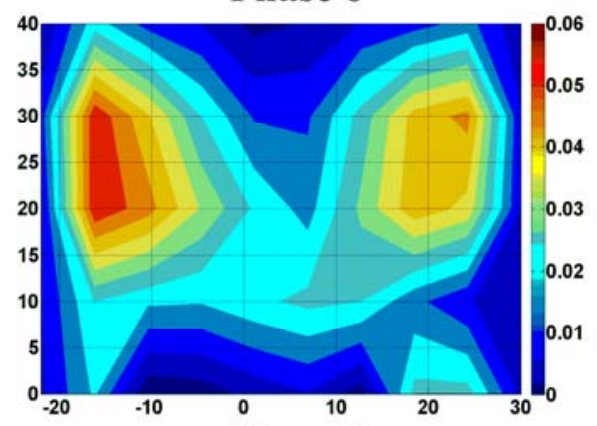

Phase 7

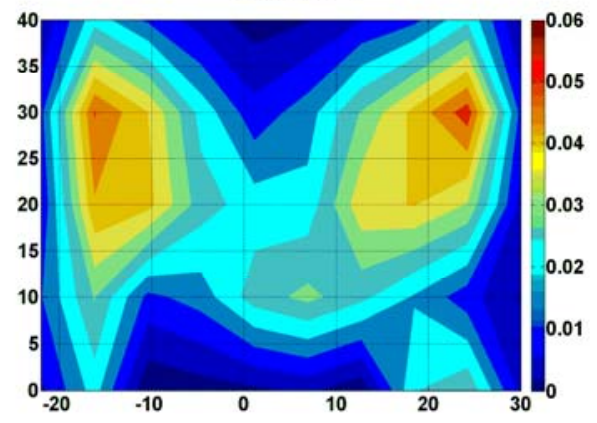

Phase 8

Figure 8 - Phase-sorted probability distribution of flame-kernel events in the 'Noisy Flame' studied in the TM-Burner. Note, unlike in the DS-burner, the measurement zone does not span the entire width of the combustor. 

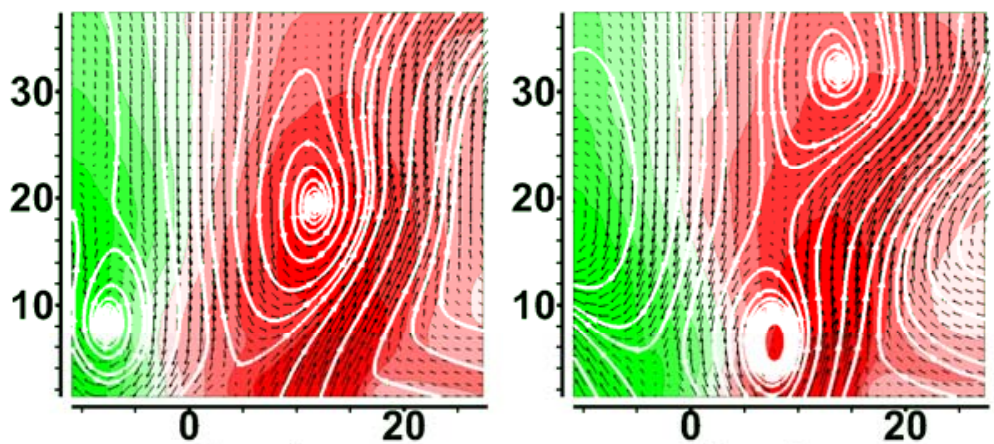

Phase 1

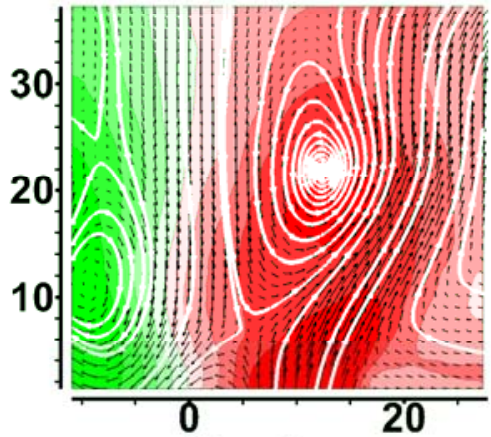

Phase 5

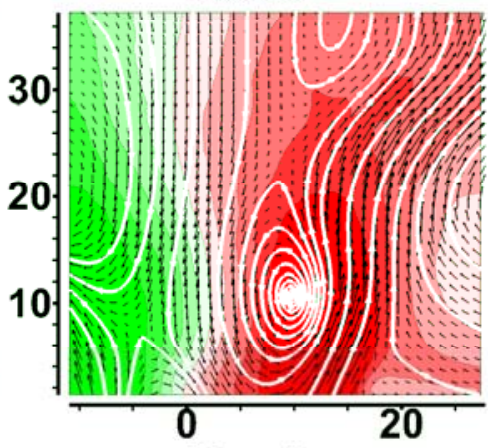

Phase 2
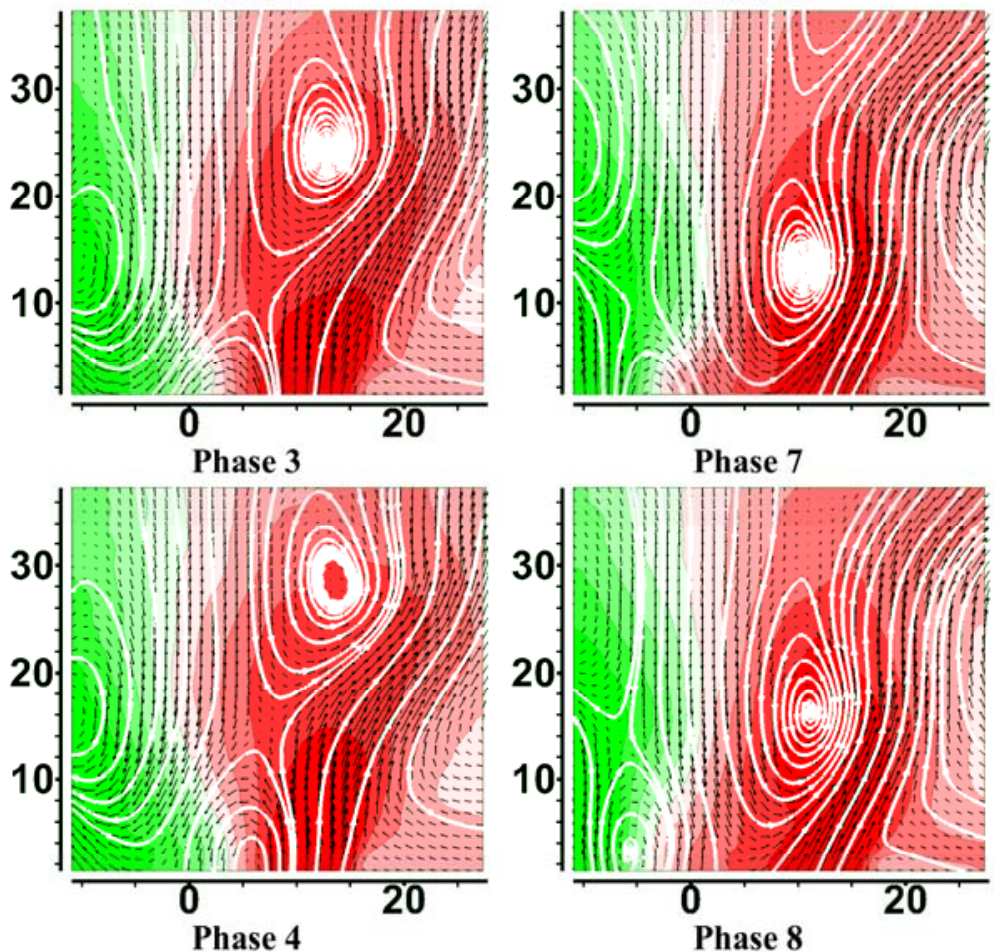

$\begin{array}{lllllllll}-15.0 & -11.3 & -7.5 & -3.8 & -0.0 & 3.8 & 7.5 & 11.3 & 15.0\end{array}$

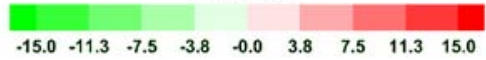

Figure 9 - Phase averaged velocity field measured in the 'Noisy flame' of the TM-Burner. Color contours represent the through-plane component of velocity, Vz. 


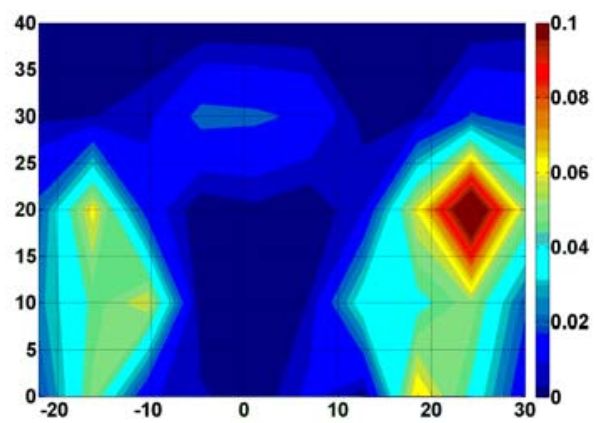

Phase 1

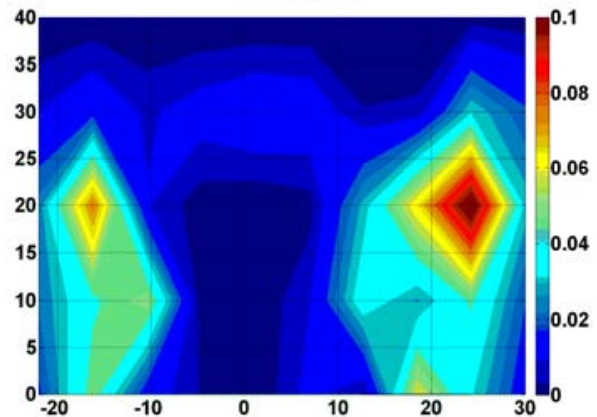

Phase 2

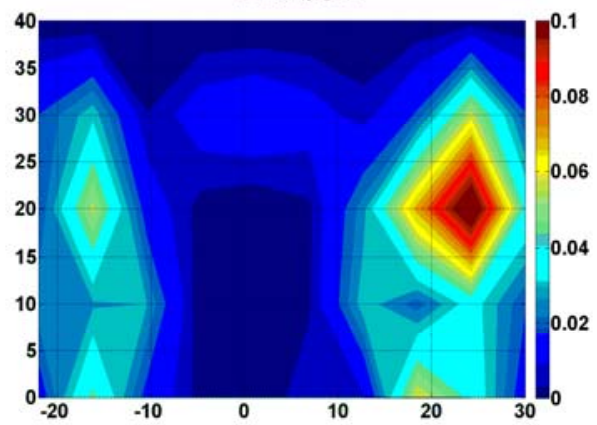

Phase 3

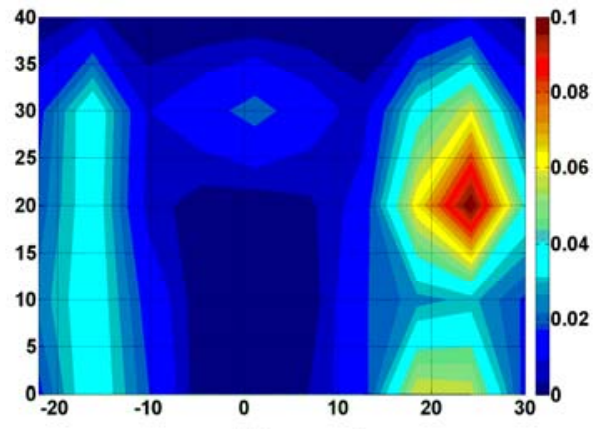

Phase 4

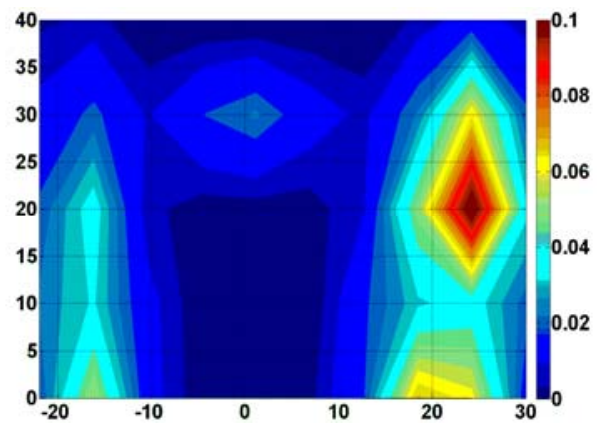

Phase 5

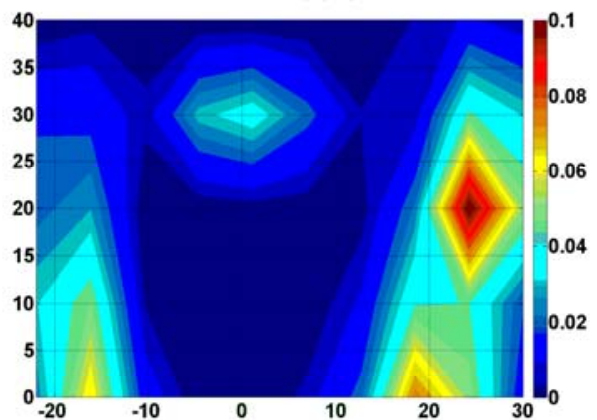

Phase 6

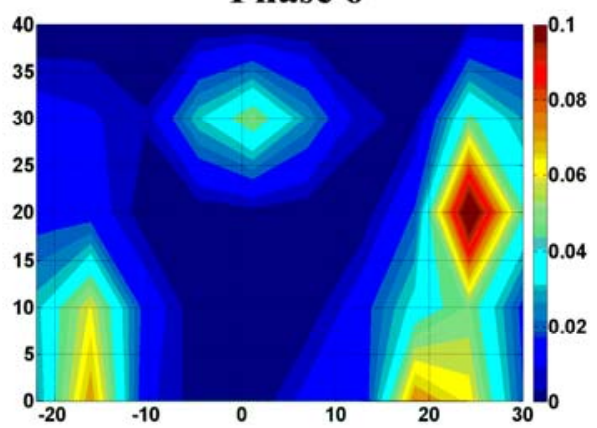

Phase 7

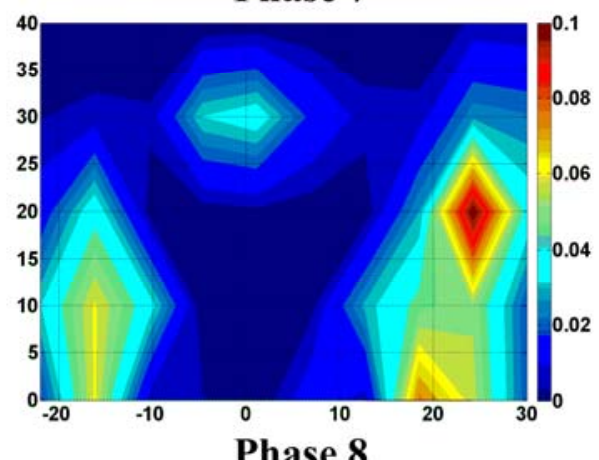

Figure 10 - Spatial distribution of flame-kernel events, phase-sorted according to the dominant velocity eigenmode, for the 'Quiet flame' studied in the TM-burner. 

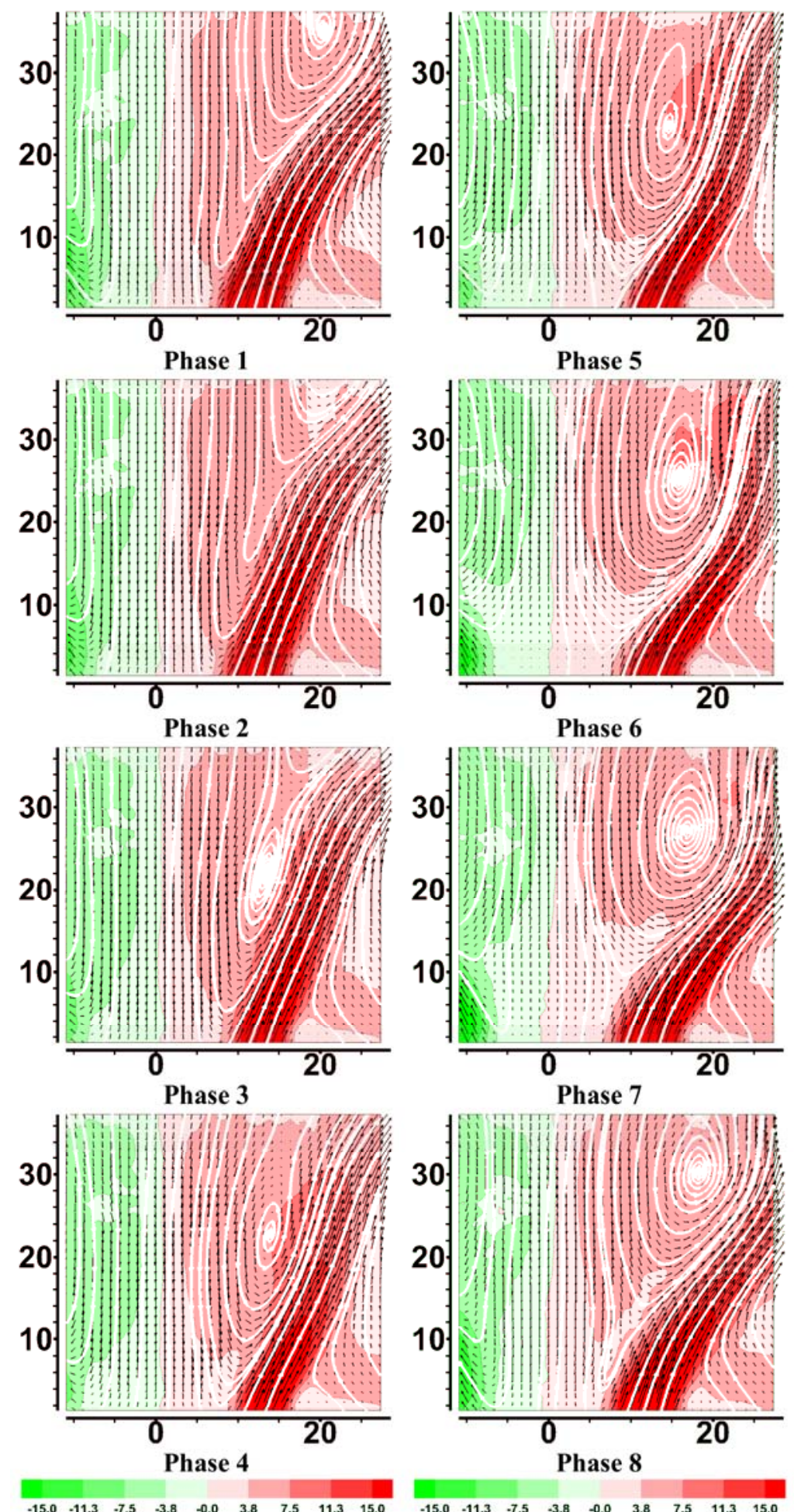

Figure 11 - Phase averaged velocity field measured in the 'Quiet flame' of the TM-burner. Color contours represent the through-plane component of velocity, Vz. 

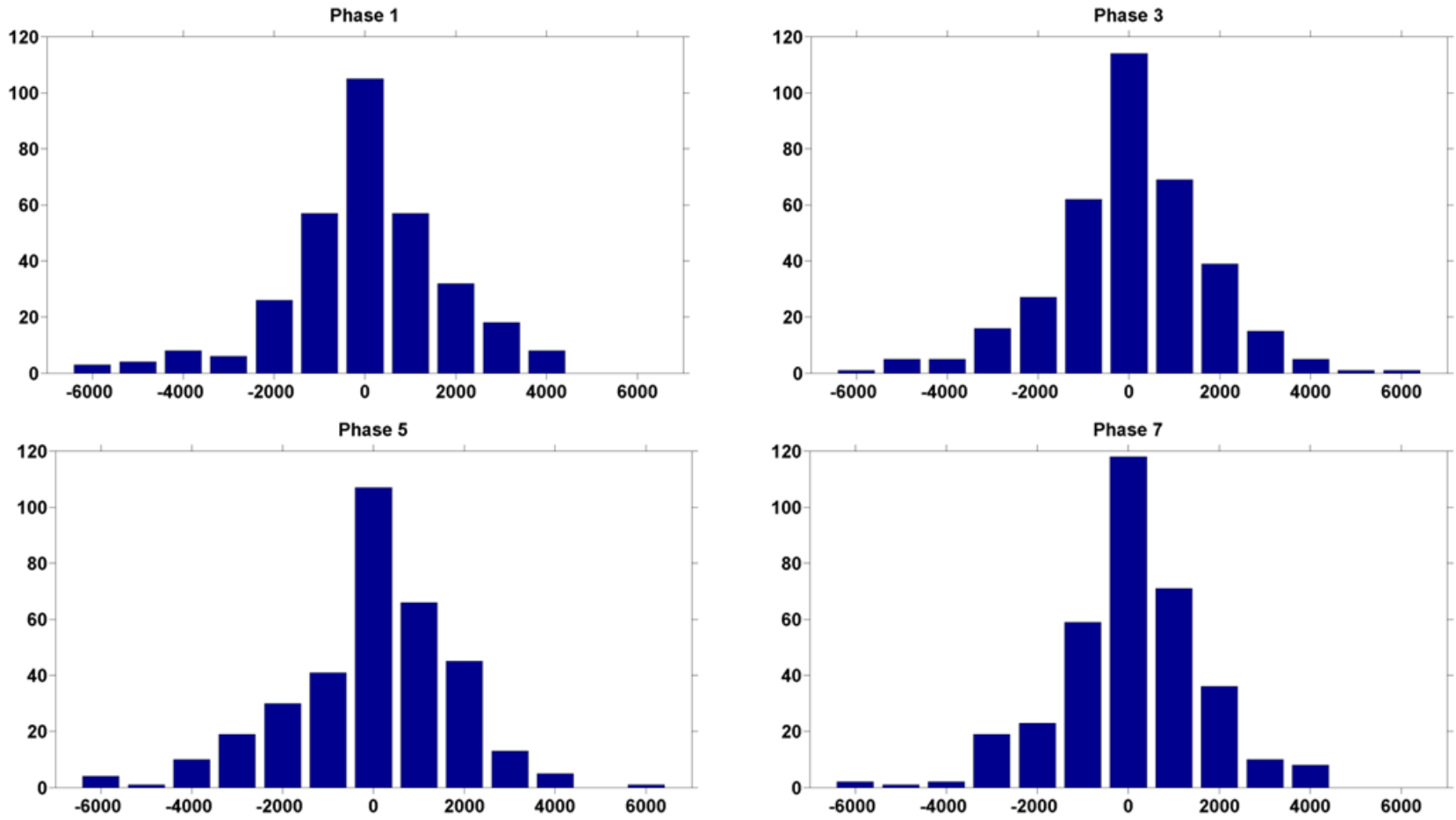

Figure 12 - Histograms of the difference between instantaneous and phase-averaged values of 2D minimum normal strain at flame kernel centroids for flames measured in the DS-burner. Units are $(1 / \mathrm{s})$. 